\title{
Magnetic Evolution and Temperature Variation in a Coronal Hole
}

\author{
Jun Zhang ${ }^{1}$, Guiping Zhou ${ }^{1}$, Jingxiu Wang ${ }^{1}$ and Haimin Wang ${ }^{2}$
}

\begin{abstract}
We have explored the magnetic flux evolution and temperature variation in a coronal-hole region, using Big Bear Solar Observatory (BBSO) deep magnetograms and SOHO/EIT images observed from 2005 October 10 to 14. For comparison, we also investigated a neighboring quiet region of the Sun. The coronal hole evolved from its mature stage to its disappearance during the observing period. We have obtained the following results: (1) When the coronal hole was well developed on October 10, about $60 \%$ of the magnetic flux was positive. The EUV brightness was 420 counts pixel $^{-1}$, and the coronal temperature, estimated from the line ratio of the EIT $195 \AA$ and $171 \AA$ images, was $1.07 \mathrm{MK}$. (2) On October 14, when the coronal hole had almost disappeared, $51 \%$ of the magnetic flux was positive, the EUV radiance was 530 counts pixel $^{-1}$, and the temperature was 1.10 MK. (3) In the neighboring quiet region, the fraction of positive flux varied between 0.49 and 0.47 . The EUV brightness displayed an irregular variation, with a mean value of 870 counts pixel $^{-1}$. The temperature was almost constant at $1.11 \mathrm{MK}$ during the five-day observation. Our results demonstrate that in a coronal hole less imbalance of the magnetic flux in opposite polarities leads to stronger EUV brightness and higher coronal temperatures.
\end{abstract}

Subject headings: Sun: corona —Sun: magnetic fields —Sun: UV radiation

\section{INTRODUCTION}

Coronal holes are cool, low-density regions observed both at low latitudes and at the polar regions of the Sun (Chiuderi Drago et al. 1999). They were first observed in white light by Waldmeier (1951), on X-ray plates by Underwood \& Muney (1967), and on EUV line

\footnotetext{
${ }^{1}$ National Astronomical Observatories, Chinese Academy of Sciences, Beijing 100012, China; wjx@ourstar.bao.ac.cn, zhougp@ourstar.bao.ac.cn, zjun@ourstar.bao.ac.cn

${ }^{2}$ Big Bear Solar Observatory, New Jersey Institute of Technology, Big Bear City, CA 92314; haimin@flare.njit.edu
} 
spectroheliograms by Reeves \& Parkinson (1970). Their predominantly unipolar magnetic fields are open to the interplanetary space (Bohlin 1977), giving rise to high-speed solar-wind streams that can lead to geomagnetic storms (Krieger \& Timothy 1973). There are three broad categories of coronal holes: polar, non-polar and transient ones (Harvey \& Recely 2002). It has been suggested that magnetic reconnection must occur continuously at the boundary of coronal holes in order to maintain the coronal-hole integrity (Kahler \& Hudson 2002). The fast solar wind starts flowing out in magnetic funnels at heights between $5000 \mathrm{~km}$ and $20000 \mathrm{~km}$ above the photosphere (Tu et al. 2005).

The electron temperature is clearly an important parameter in the corona. A detailed assessment of observations in coronal holes and the deduced temperatures was published by Habbal et al. (1993). Electron temperatures in the corona can be measured with the help of a magnesium line ratio of a temperature-sensitive pair (cf. Wilhelm 2006), with the assumption that the density and temperature of the gas from which spectral lines are emitted are constant along the line of sight (Habbal et al. 1993). It should be mentioned that temperatures in the inner corona cannot be accurately derived as there are many sources of uncertainty such as instrument calibration, line-of-sight effects, departure from ionization balance, and inaccuracies of the atomic data.

In recent years, the coronal temperatures have been intensively studied since space observations are ascertained from the YOHKOH and SOHO missions (Hara et al. 1992, 1994; Moses et al. 1997). Using the two SOHO spectrometers, CDS and SUMER, electron temperatures were measured as a function of height above the limb in a polar coronal hole (David et al. 1998; Wilhelm et al. 1998). Doschek \& Laming (2000) concluded that the emission line ratio increase in a polar coronal hole was primarily due to an increase of the electron temperature with height. Marsch et al. (2000) found that the hydrogen temperature increased only slightly from $1 \times 10^{5} \mathrm{~K}$ to $2 \times 10^{5} \mathrm{~K}$ in the height range from $12000 \mathrm{~km}$ to $18000 \mathrm{~km}$, and Stucki et al. (2000) presented that with increasing formation temperature, spectral lines displayed on average an increasingly stronger blueshift in coronal holes relative to the quiet Sun at equal heliospheric angle. Furthermore, Xia et al. (2004) reported that the bases of coronal holes seen in chromospheric spectral lines with relatively low formation temperatures displayed similar properties as normal quiet-Sun regions. More recently, Wilhelm (2006) reported that, in a polar coronal hole region, the electron temperatures in plumes are $7.5 \times 10^{5} \mathrm{~K}$ and $1.13 \times 10^{6} \mathrm{~K}$ in inter-plume regions, in the height of $45 \mathrm{Mm}$ above the limb.

In this Letter, we study the magnetic evolution, the EUV brightness changes, and the coronal temperature variations in a coronal hole and an adjacent quiet region from 2005 October 10 to 14 . Initially, the coronal hole was well developed. At the end, the coronal hole 
had almost disappeared. Combining deep magnetograms (with a noise level of $2 \mathrm{G}$ ) from Big Bear Solar Observatory (BBSO) with SOHO/EIT observations, we unravel the nature of the different magnetic properties and temperature variations in this coronal hole and the quiet region.

\section{OBSERVATIONS}

From 2005 October 10 to 14, the observational target of BBSO was a coronal hole and a neighboring quiet region. The target was very close to the equator, centered at $\mathrm{S} 3^{\circ} \mathrm{E} 28^{\circ}$ on the 10th, and at $\mathrm{S}^{\circ} \mathrm{W} 25^{\circ}$ on the 14 th. The magnetogram was obtained using the digital vector magnetograph (DVMG) system mounted on the $25 \mathrm{~cm}$ refractor. The DVMG system uses liquid crystal, a Zeiss filter, and a 12 bit digital camera so that one can accurately measure small intranetwork magnetic elements on the order of $2 \mathrm{G}$. The temporal resolution is $90 \mathrm{~s}$, and the field of view is $300^{\prime \prime} \times 300^{\prime \prime}\left(0^{\prime \prime} .6 \mathrm{pixel}^{-1}\right)$. The EUV observations were obtained by the Extreme Ultraviolet Imaging Telescope (EIT) on board of $S O H O$. The instrument generally observes full-disk EUV images in the coronal $171 \AA$ (Fe IX/X, $\approx 1 \mathrm{MK}), 195 \AA$ $(\mathrm{Fe} \mathrm{XII}, \approx 1.5 \mathrm{MK})$, or $284 \AA(\mathrm{Fe} \mathrm{XIV}, \approx 2 \mathrm{MK})$ passbands. A detailed description of the instrument is provided by Delaboudinière et al. (1995).

Figure 1 shows a full-disk MDI (Scherrer et al. 1995) magnetogram (top left frame), an EIT $195 \AA$ image (middle left frame) and a temperature map (bottom left frame) derived from ratio of the $195 \AA$ and $171 \AA$ images. High values of this ratio generally outline magnetically closed field regions. The coronal holes are clearly defined as dark, i.e., cool regions in the ratio image both on and off the disk (Moses et al. 1997). Three windows on the full-disk images outline the field of view of BBSO magnetograms. Half of the area is a coronal hole, and the other half a quiet region. Dashed-lines in the three frames of the right column separate the coronal hole from the quiet region.

\section{MAGNETIC FIELD EVOLUTION AND TEMPERATURE VARIATION}

Figure 2 shows BBSO magnetograms (left column) in the region marked by the small windows in Figure 1. An ephemeral region (ER1) appeared near 17:49 UTC, October 11. As ER1 is growing, another smaller ER2 appeared between the two elements of ER1 (see the magnetogram at 19:29 UTC). The negative element of the smaller ER2 merged into the larger negative element of ER1, and the positive element of ER2, canceled with the opposite polarity element of the ER1. Three hours later, ER2 disappeared. The interaction of the 
two ERs is associated with the increased EUV emission in the right column of Fig. 2. Figure 3 presents the evolution of magnetic flux density versus time in the upper panel, and in the middle panel the evolution of EUV emission. By tracking ER1, we find that it went through four phases ('P1', 'P2', 'P3', and 'P4') in its evolution. Firstly, ER1 continuously emerged for $15 \mathrm{~h}$, meanwhile its positive flux canceled with the pre-existing negative flux. At this stage, the flux emergence was dominant. In the second phase, the flux was almost stable, as seen from MDI magnetograms. The third phase began at 04:24 UTC on October 12. A new ER appeared in the area, and interacted with ER1. Eight hours later, the new ER could not be tracked any longer. At this time, the mean flux density in this area reached the highest value. Finally, low-level emergence and cancellation intermittently persisted for about one day, before ER1 faded away. There was quite a close relationship between the evolution of magnetic flux density and the variation of the EUV brightness. The coronal temperature, shown in the bottom panel of Figure 3, deduced from the ratio of the Fe XII and Fe IX/X channels, also increased in the first three phases. However in the last phase, the temperature variation did not follow that of magnetic flux density.

It is well known that coronal holes lie within a predominantly unipolar magnetic region, but the solar magnetic field is never strictly unipolar. Wang et al. (1992) found that the minority polarity flux occupied $15 \%$ to $30 \%$ of the total magnetic flux in a coronal hole. However, we have little knowledge about the magnetic flux evolution in coronal holes. Here we study the evolution of the magnetic flux and the variation of the flux imbalance in a coronal hole during its decaying stage, and compare them with those in a quiet region. The top panel of Figure 4 displays the evolution of the total unsigned flux, measured from BBSO magnetic field data, in the coronal hole and the quiet region. We notice that during the five-day observations, the total fluxes in both regions were almost stable within the standard uncertainty margin. This result is confirmed by seeing-free MDI magnetic field data. The fraction of the positive flux to the total flux in the two regions is presented in the next panel. About $58 \%$ of the magnetic fields was positive in the coronal hole on October 10, the day when it was well developed. During the period in which the coronal hole gradually disappeared, the fraction of the positive flux decreased accordingly. The fraction decreased to $51 \%$ at the end, indicating that the fluxes of the positive and negative polarities were nearly balanced. In contrast to the coronal-hole region, we found that the ratio between positive and negative fluxes in the quiet region did not change significantly. During the fiveday observing period, the fraction of positive flux varied between $49 \%$ and $47 \%$, indicating that the fluxes of the two polarities are approximated balanced all the time.

The EUV brightness and temperature variations of the two regions are presented in the lower panels of Figure 4. In the quiet region, the EUV radiation fluctuates without a trend. The mean value is 870 counts pixel $^{-1}$. The coronal temperature is almost stable 
at a level of $1.11 \mathrm{MK}$ during the five days. In the coronal hole, although the brightness also fluctuates, there is a clear ascending trend. From October 11 to 14, the mean EUV brightness increased from 420 to 530 counts pixel $^{-1}$, i.e. a relative increase of about $26 \%$. The derived temperature also increased. On October 10 and 11, the temperature was about $1.07 \mathrm{MK}$, and on October 14 it reached 1.10 MK.

\section{CONCLUSIONS AND DISCUSSIONS}

In this Letter, we probe the relationship between the magnetic field evolution, the EUV brightness changes and the coronal temperature variations in a coronal hole, and compare it with that of a neighboring quiet region. In the coronal hole, $58 \%$ of the magnetic field was positive while the hole was well developed. When it almost disappeared, the fraction of the positive flux decreased to $51 \%$. This means that one of the signatures of decay of a coronal hole is the disappearance of the flux imbalance. Although the EUV emission fluctuated when the coronal hole was decaying, it clearly had an increasing trend. From October 11 to 14, the EUV increased from 420 to 530 counts pixel $^{-1}$, a relative increase of about $26 \%$. The coronal temperature, deduced from the Fe IX/X and Fe XII line ratio, also increased. When the hole was well developed, the temperature was about $1.07 \mathrm{MK}$, and when the coronal hole almost disappeared, the temperature reached 1.10 MK. In the quiet region, the magnetic fluxes in both polarities were always approximately balanced. The EUV radiation fluctuates slightly with a mean of 870 counts pixel $^{-1}$, and the temperature was stable at a level of 1.11 MK. By using a similar line-ratio method, Moses et al. (1997) presented a full-disk temperature map. From the map, we deduced a coronal temperature range was from 1.00 $\mathrm{MK}$ to $1.10 \mathrm{MK}$ in coronal holes, and from 1.10 MK to $1.20 \mathrm{MK}$ in quiet regions. Our coronal temperatures are basically consistent with those of Moses et al. (1997).

By checking the flux transport across the coronal-hole boundary for this region with MDI data, we do not see a significant migration of positive or negative fluxes. The only explanation of the magnetic field evolution then is that positive network flux canceled with "hidden" negative intranetwork (IN) flux, i.e., the magnetic flux which was too weak to be detected. Zhang et al. (2006) found that the net IN flux is opposite to that of network flux. The ephemeral regions, in turn, refurbished the missing flux. If we assume that at the mature stage of a coronal hole, there are ten units of total flux, about six unit are positive and four negative. When it evolved to the decayed stage, two positive units disappeared due to cancellation with invisible IN flux. Meanwhile ephemeral regions provide one additional unit of flux for each of the two polarities. Consequently, five units each for the positive and negative fluxes would be detected. 
We would like to thank the referee, Dr. Klaus Wilhelm, for valuable comments and useful suggestions. The authors are indebted to the SOHO/EIT, MDI and BBSO teams for providing the data. $S O H O$ is a project of international cooperation between ESA and NASA. This work is supported by the National Natural Science Foundations of China (G40674081, 10573025 and 10233050), the National Basic Research Program of China under grant G2006CB806303, and two US NASA grants (NNG0-6GI19G and NNG0-4GG21G).

\section{REFERENCES}

Bohlin, J. D. 1977, Sol. Phys., 51, 377

Chiuderi Drago, F., Landi, E., Fludra, A., \& Kerdraon, A. 1999, A\&A, 348, 261

David, C., Gabriel, A. H., Bely-Dubau, F. et al. 1998, A\&A, 336, L90

Delaboudinière, J-P., Artzner, G. F., Brunaud, J. et al. 1995, Sol. Phys., 162, 291

Doschek, G. A., \& Laming, J. M. 2000, ApJ, 539, L71

Habbal, S. R., Esser, R., \& Arndt, M. B. 1993, ApJ, 413, 435

Hara, H., Tsuneta, S., Lemen, J. R. et al. 1992, PASJ, 44, L135

Hara, H., Tsuneta, S., Acton, L. W. et al. 1994, PASJ, 46, 943

Harvey, K. L., \& Recely, F. 2002, Sol. Phys., 211, 31

Kahler, S. W., \& Hudson, H. S. 2002, ApJ, 574, 467

Krieger, A. S., \& Timothy, A. F. 1973, Sol. Phys., 29, 505

Marsch, E., Tu, C., \& Wilhelm, K. 2000, A\&A, 359, 381

Moses, D., Clette, F., Delaboudinière, J-P. et al. 1997, Sol. Phys., 175, 571

Reeves, E. M., \& Parkinson, W. H. 1970, ApJS, 21, 1

Scherrer, P. H., Bogart, R. S., Bush, R. I. et al. 1995, Sol. Phys., 162, 129

Stucki, K., Solanki, S. K., Schühle, U. et al. 2000, A\&A, 363, 1145

Tu, C., Zhou, C., Marsch, E. et al. 2005, Science, 308, 519

Underwood, J. H., \& Muney, W. S. 1967, Sol. Phys., 1, 129 
Waldmeier, M. 1951, Z. Astrophys., 30, 1

Wang, J., Wang, H., \& Shi, Z. 1992, ASP Conference Series, 27, 108

Wilhelm, K. 2006, A\&A, 455, 697

Wilhelm, K., Marsch, E., Dwivedi, B. N. et al. 1998, ApJ, 500, 1023

Xia, L. D., Marsch, E., \& Wilhelm, K. 2004, A\&A, 424, 1025

Zhang, J., Ma, J., \& Wang, H. 2006, ApJ, 649, 464 
Fig. 1. - A full-disk magnetogram from SOHO/MDI (top left), an EIT $195 \AA$ image from SOHO/EIT (middle left), and a temperature map (bottom left) of the ratio from the EIT $195 \AA$ and $171 \AA$ channels. Three windows in the three full-disk images outline the field of view of BBSO magnetograms. The small windows in the right column denote a sub-area where ephemeral regions appear (see Fig. 2) 
Fig. 2.- Time sequences of BBSO magnetograms (left) and EIT $195 \AA$ images (right) on 2005 October 11. The arrows show two pairs of ephemeral regions, and the ellipses encircle their areas. The field of view is about $20^{\prime \prime} \times 20^{\prime \prime}$. 
Fig. 3.- Magnetic flux density evolution (top) measured from MDI magnetic field data versus time in the field of view of Fig. 2. The middle and bottom panels display the corresponding EUV brightness change and temperature variation, respectively. Three vertical lines separate the four phases ('P1', 'P2', 'P3', and 'P4') of ER1. 
Fig. 4.- The evolution of the total magnetic flux (top) and of the positive magnetic flux fraction (upper middle) measured from BBSO magnetograms versus time in the coronal hole and the quiet region shown in the field of view of the right column of Fig. 1. The lower panels display the variations of the EUV emission and temperature changes. 